\title{
Auxin signaling and regulated protein degradation
}

\author{
Nihal Dharmasiri and Mark Estelle
}

Department of Biology, Indiana University, Bloomington, IN 47405, USA

\begin{abstract}
Auxin regulates many aspects of plant growth and development. Auxin is known to regulate gene expression through degradation of the AUX/IAA proteins, short-lived nuclear proteins that regulate gene transcription. The AUX/IAA proteins are degraded through the action of a ubiquitin protein ligase called $\mathrm{SCF}^{\mathrm{TIR} 1}$. Auxin promotes the interaction between AUX/IAA proteins and SCF ${ }^{\mathrm{TIR} 1}$ in a soluble extract, suggesting that the auxin receptor is a soluble factor. These studies also indicate that the auxin-induced AUX/IAA-SCF ${ }^{\text {TIR1 }}$ interaction does not depend on phosphorylation or hydroxylation of AUX/IAA proteins. Although the mechanism of auxin-induced AUX/IAA$\mathrm{SCF}^{\mathrm{TIR} 1}$ interaction is not yet clear, auxin might induce a modification in TIR1, AUX/IAA proteins or an adaptor protein that is required for the interaction.
\end{abstract}

Auxin plays a pivotal role in many processes throughout the plant life cycle. These include embryogenesis, lateral root development, vascular differentiation, apical dominance, tropic responses and flower development. Auxin was first discovered decades ago [1]. In spite of the tremendous amount of information that has accumulated since then, the auxin signaling pathways have not been fully elucidated. Nevertheless, through the combined application of genetic, molecular and biochemical methods, we are beginning to understand some aspects of auxin action. In this article, we discuss recent developments in the study of auxin signaling, particularly focusing on auxin-regulated transcription.

\section{Auxin modulates gene transcription}

It has long been known that auxin stimulates the transcription of a set of genes called primary auxin responsive genes. The known primary genes include three gene families called the AUX/IAA, GH3 and SAUR (small auxin-up RNA) families [2]. The AUX/IAA proteins are short-lived nuclear proteins that function as transcriptional regulators [3]. These proteins do not interact directly with DNA but exert their regulatory activity through another group of proteins called auxin response factors (ARFs). There are at least 29 AUX/IAA genes in the Arabidopsis genome. Most of the AUX/IAA proteins share four conserved domains, designated domains I to IV. Domains III and IV are located in the C-terminal half of the protein and are involved in homo- and heterodimerization

Corresponding author: Mark Estelle (maestell@indiana.edu). with other AUX/IAA proteins and heterodimerization with ARFs that also share domains III and IV (also called the CTD or C-terminal domain). Additionally, ARFs contain an $\mathrm{N}$-terminal DNA binding domain (DBD) [4]. There are 23 $\mathrm{ARF}$ genes in the Arabidopsis genome and all but two (ARF3 / ETTIN and ARF17) contain the CTD region [5,6]. ARFs bind to conserved DNA sequences (TGTCTC) called auxin-response elements (AuxREs) in the promoter regions of primary/early auxin response genes [6]. ARFs can act as either transcriptional activators or repressors depending on the nature of their middle region (MR) domain [7,8]. The ARFs with a Q-rich MR function as activators, whereas other ARFs with a P/S/T-rich MR function as transcriptional repressors [5]. Thus, AUX/IAA proteins, which repress the activity of ARFs, function as negative or positive regulators of gene transcription. Contrary to the earlier suggestion that the ARFs act as dimers, new data suggest that they can act as monomers to regulate gene transcription, and dimerization of ARFs might potentiate their effects $[6,9,10]$.

The AUX/IAA proteins generally have a high turnover rate. The half-life of these proteins in wild-type Arabidopsis seedlings ranges from $\sim 10 \mathrm{~min}$ to $\sim 80 \mathrm{~min}$, depending on the protein [11-13]. This short half-life can be extended severalfold by treatment with proteasome inhibitors such as MG115 and MG132, indicating that the degradation of the AUX/IAA proteins is associated with the proteasome pathway [11].

\section{Auxin signaling is mediated through the ubiquitin- proteasome pathway}

In Arabidopsis, the characterization of the auxin resistant mutants axr1 and tir1 led to the discovery that the ubiquitin-proteasome pathway is involved in auxin response $[12,14,15]$. Ubiquitin-mediated protein degradation has emerged as a vital process that regulates the growth and development of eukaryotic organisms. Proteins that are destined to be destroyed are tagged with a polyubiquitin chain by a cascade of reactions involving three enzymes, known as the ubiquitin activating enzyme (E1), ubiquitin conjugating enzyme (E2) and ubiquitin protein ligase (E3). Ubiquitinated proteins are recognized and degraded by the $26 \mathrm{~S}$ proteasome, a multiprotein complex comprising a $20 \mathrm{~S}$ core unit and two $19 \mathrm{~S}$ regulatory particles. The ubiquitination process begins with the E1 forming a thiol-ester linkage with a ubiquitin molecule. The ubiquitin molecule is then transferred to the E2 and finally covalently attached to the target protein 
with the help of an E3 ubiquitin ligase. This process is repeated until several ubiquitin moieties are attached to the target protein as a polyubiquitin chain. Generally, the specificity of the pathway is determined by the E3. Thus, organisms have many E3 s that allow the ubiquitination of a diverse array of proteins. Currently, several groups of E3 $\mathrm{s}$ have been identified that differ in their subunit organization and/or mechanism of ubiquitin transfer [16]. In this article, we focus on the SCF (Skp1-Cullin-F-box) type of E3, which is composed of four primary subunits: Cullin1/Cdc53, Skp1/ASK1, Rbx1/ROC1/Hrt1 and an F-box protein.

The TIR1 gene encodes an F-box protein that contains an N-terminal F-box region and C-terminal leucine rich repeats. Generally, F-box proteins interact with the CUL1-RBX1 subcomplex through the ASK1 or ASK2 proteins. TIR1 immunoprecipitates with CUL1, ASK1 and RBX1, indicating that these subunits form an SCF ${ }^{\mathrm{TIR} 1}$ complex [15,17]. In line with this, the tir1, ask1 and axr6 mutants are resistant to auxin $[15,18]$. The AXR6 gene encodes the Arabidopsis CULLIN1 protein (CUL1) and is required for normal embryonic and postembryonic development [18]. In Arabidopsis, two independent axr6 mutations have been identified and both alleles result in a substitution of Phe111 with either valine (axr6-1) or isoleucine (axr6-2). Interestingly, this phenylalanine residue lies in the region that interacts with SKP1 in the solved structure of human $\mathrm{SCF}^{\mathrm{SKP} 2}$. Further, biochemical studies indicate that interaction between CUL1 and ASK1 is impaired in axr6 mutants [18].

\section{AUX/IAA proteins are degraded through SCF ${ }^{\text {TIR1 }}$}

The rapid turnover of the AUX/IAA proteins and their stabilization by proteasome inhibitors suggested that AUX/IAAs might be $\mathrm{SCF}^{\mathrm{TIR} 1}$ substrates $[11,12]$. In fact, two AUX/IAA proteins (IAA7 and IAA17) interact with $\mathrm{SCF}^{\mathrm{TIR} 1}$ in pull-down assays, indicating that AUX/IAA proteins are substrates for $\mathrm{SCF}^{\mathrm{TIR} 1}$ [12]. This interaction is promoted by auxin in a concentration dependant manner in planta, and the effect of auxin can be seen as early as 5 min after auxin application [12]. Genetic studies have identified several gain-of-function mutants in AUX/ IAA genes. Interestingly, all of the mutations are found in domain II of the affected proteins [19-22]. When a domain II peptide containing 13 amino acids is fused to the firefly luciferase reporter protein, the recombinant protein is rapidly degraded in a transient assay using tobacco protoplasts or in stable Arabidopsis transgenic plants $[11,23]$. Domain II encompasses the conserved amino acid residues GWPPV. When amino acid substitutions corresponding to those in known AUX/IAA mutants were introduced to these conserved residues, the mutations stabilized the recombinant protein [11]. This suggests that domain II is a 'degron' that is essential and sufficient for $\mathrm{SCF}^{\mathrm{TIR} 1}$-mediated AUX/IAA degradation. So far, several AUX/IAA proteins, including AXR2/IAA7, AXR3/IAA17 and SHY2/IAA3, have been shown to interact with the $\mathrm{SCF}^{\mathrm{TIR} 1}[12,24]$. Thus, it is possible that most, perhaps all, of the AUX/IAA proteins interact with the SCF ${ }^{\mathrm{TIR} 1}$ and undergo subsequent ubiquitination and degradation.

\section{RUB modification of CUL1 is essential for normal auxin response}

Genetic evidence suggests that modification of CUL1 by a ubiquitin-related protein called RUB1 (related to ubiquitin 1) is essential for normal auxin response. RUB is a conserved protein in eukaryotes (NEDD8 in human and fission yeast) and, in Arabidopsis, there are three RUB genes. RUB1 is covalently attached to a lysine residue in the C-terminal region of CUL1. Like ubiquitin, RUB attachment involves an E1 and E2 enzyme but unlike the ubiquitin E1, the RUB E1 enzyme is a heterodimer. In Arabidopsis, the two E1 subunits are AXR1 and ECR1. The Arabidopsis RUB E2 is called RCE (RUB-conjugating enzyme) and the Arabidopsis genome contains two RCE genes, RCE1 and RCE2 [14]. As for the RUB E3, recent studies suggest that the RBX1 protein functions as the E3 for RUB conjugation to CUL1 in Arabidopsis and budding yeast, and to human Cul2 [17,25,26]. The loss-of-function mutants axr 1 and rce 1 result in reduced CUL1 modification and consequent growth and development defects. The axr 1 rce 1 double mutant has a strong reduction in CUL1 modification and displays a severe seedling-lethal phenotype similar to the monopteros $(\mathrm{mp})$ and bodenlos (bdl) mutants [25]. The $M P$ gene encodes the ARF5 transcription factor and the $B D L$ gene encodes the IAA12 protein. A loss-of-function mutation in ARF5 $(\mathrm{mp})$ or a gain-of-function mutation in IAA12 $(b d l)$ affects the formation of hypophysis and other basal structures in the embryo [27]. The axr6 mutants also have reduced level of RUB-CUL1. The exact reason for accumulation of unmodified CUL1 in the axr6 mutants is not clear because the AXR6-1 and AXR6-2 proteins appear to be efficient substrates for RUB modification in vitro. One possibility is that the mutant proteins are better substrates for RUB deconjugation than the wild-type proteins. Alternatively, CUL1 might need to be in a complex with ASK1-F-boxprotein, and perhaps other proteins, for efficient RUB conjugation in vivo [18].

\section{SCF activity might be regulated at different levels}

The AUX/IAA proteins are degraded via $\mathrm{SCF}^{\mathrm{TIR} 1}$ in response to auxin (Figure 1). How is $\mathrm{SCF}^{\mathrm{TIR} 1}$ activity regulated? None of the genes encoding proteins in $\mathrm{SCF}^{\mathrm{TIR} 1}$ or the RUB pathway are known to be regulated at the transcriptional level by auxin. Although post-transcriptional or post-translational regulation of $\mathrm{SCF}^{\mathrm{TIR} 1}$ and/or the RUB pathway proteins is a possibility, no such activity has been identified. However, it has been found that some F-box proteins are generally unstable owing to autoubiquitination $[28,29]$. Although there are no data available on the stability of TIR1, it is possible that the TIR1 protein is degraded through autoubiquitination in the absence of auxin (Figure 1).

Another possible way to regulate AUX/IAA degradation is altering SCF activity through CUL1 modification. What is the function of CUL1-RUB modification and how does RUB conjugation affect the auxin response? The answers to these questions are not yet clear. Some studies report that CUL1 modification increases the activity of the SCF in vitro [30-32]. How this occurs is not clear because CUL1 modification affects neither assembly nor stability of the 


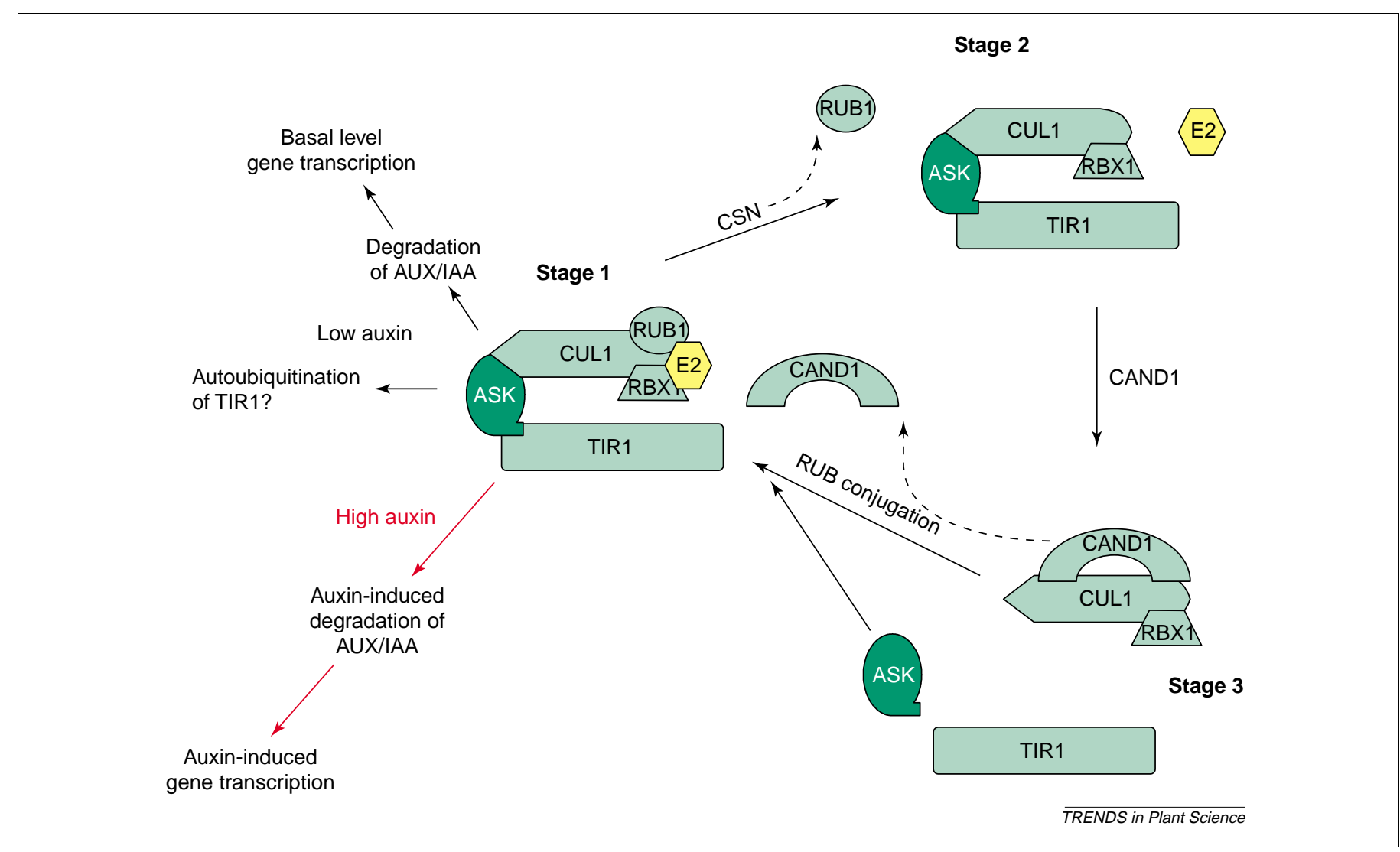

Figure 1. Regulation of SCF ${ }^{\mathrm{TIR} 1}$ assembly and activity through RUB1 conjugation and deconjugation. The functional SCF ${ }^{\mathrm{TIR} 1}$ is represented by Stage 1 . AUX/IAA proteins interact with TIR1 and are ubiquitinated in the presence of auxin. Removal of RUB by the COP9 signalosome (CSN) will release E2 (Stage 2) and stimulate the association of CAND1. This will dissociate ASK1 and TIR1 from the SCF ${ }^{\text {TR1 }}$ complex (Stage 3). Association of ASK1-TIR1 with CUL1 will dislodge CAND1, allowing CUL1 to be modified by RUB1. At low auxin levels, degradation of AUX/IAA proteins might occur at a basal rate. At high auxin levels, enhanced interaction between AUX/IAAs and TIR1 will result in increased AUX/IAA degradation and increased transcription of auxin-regulated genes.

SCF. However, it has been suggested that NEDD8/RUB1 modification might enhance the affinity of the cullin for the ubiquitin E2 [33,34]. Thus, one function of the modification might be to recruit the $\mathrm{E} 2$ to the SCF complex (Figure 1). If CUL1 modification enhances the activity of the SCF, a decrease in modification should result in a reduction in SCF activity. Consistent with this, the auxin response mutants axr1-12, rce1 and axr6 exhibit a defect in AUX/ IAA degradation [12,18,25]. In Arabidopsis, RBX1 overexpression and mutations that affect the COP9 signalosome (CSN) result in enhanced CUL1 modification. Surprisingly these lines are also defective in both AUX/ IAA degradation and auxin response, suggesting that RUB conjugation and deconjugation are both important for SCF function and normal auxin response [17,35].

How does the cell regulate the dynamic process of RUB conjugation and deconjugation? Recent studies of another protein complex called the CSN might provide part of the answer. The CSN has been found to possess an isopeptidase activity that removes RUB/NEDD8 from cullin [35]. Addition of the purified CSN complex to an in vitro $\mathrm{p} 27^{\text {kip } 1}$ ubiquitination system inhibits p27 ubiquitination and degradation in a NEDDylation-dependent manner [36]. In Arabidopsis, a reduction in the level of the CSN results in increased levels of modified CUL1 and growth defects similar to the axr 1 and rce 1 mutants [35,37]. Furthermore, a defect in the CSN results in stabilization of the AUX/IAA proteins and a reduction in auxin induced AUX/IAA gene expression [35].

Recent studies in animal systems have implicated another protein in the regulation of SCF function. Several groups demonstrated that the TATA-binding proteininteracting protein $(120 \mathrm{kDa})$ (TIP120A or CAND1) preferentially interacts with unmodified CUL1 [38-40] (Figure 1). This interaction dissociates SKP1 from CUL1, thus inhibiting SCF function. In cultured mammalian cells, CAND1 knockdown using small interfering RNAs results in a moderate increase in the levels of the $\mathrm{SCF}^{\mathrm{SKP} 2}$ substrate and $\mathrm{p} 27$, and increases the affinity of SKP1-SKP2 for CUL1. CAND1 knockdown also decreases the abundance of the F-box protein SKP2. This effect might be due to increased assembly of $\mathrm{SCF}^{\mathrm{SKP} 2}$ and a subsequent increase in SKP2 autoubiquitination [40]. Interactions of unmodified CUL1 with CAND1 and SKP1 are mutually exclusive, suggesting that CAND1 negatively regulates SCF assembly. By contrast, RUB/NEDD8 modification promotes the dissociation of CAND1 from CUL1 and thus promotes SCF assembly [39] (Figure 1). CUL1 interacts with CAND1 through its N-terminal and C-terminal regions. Substitution of the lysine 720 that is the site of RUB modification with arginine (K720R) slightly reduced the interaction with CAND1, suggesting that the interaction might involve conserved K720. The Arabidopsis genome contains a CAND1 homolog 
(At2 g02560). The function of this gene in SCF regulation is currently being investigated (W. Gray, pers. commun.).

A model explaining how $\mathrm{SCF}^{\mathrm{TIR} 1}$ might be regulated by cycles of RUB conjugation and deconjugation is presented in Figure 1. According to this model, the functional $\mathrm{SCF}^{\mathrm{TIR} 1}$ is represented by stage 1 , in which CUL1 is modified by RUB1. The removal of RUB1 by CSN causes ubiquitin E2 to be displaced and promotes the association of CAND1 with CUL1 (stages 2 and 3). The CAND1-CUL1 interaction dislodges ASK1 and TIR1 from the complex (stage 3). Reassociation of ASK1 with CUL1 and/or RUB1 modification of CUL1 dislodges CAND1 from CUL1, allowing the reassembly of the $\mathrm{SCF}^{\mathrm{TIR} 1}$. Once assembled, $\mathrm{SCF}^{\mathrm{TIR} 1}$ interacts with the AUX/IAA proteins in an auxindependent manner, resulting in their ubiquitination and degradation. At low auxin levels, the AUX/IAA proteins are degraded at a basal level. When the auxin concentration is increased, enhanced interaction between AUX/ IAA proteins and TIR1 will promote the degradation of AUX/IAA proteins, resulting in an increase in auxinregulated transcription.

\section{Auxin action can be mimicked in a cell-free system}

Auxin promotes the interaction between AUX/IAA proteins and $\mathrm{SCF}^{\mathrm{TIR} 1}$ when Arabidopsis seedlings are treated with exogenous auxin. This auxin-induced interaction is evident as early as 5 min after auxin application [12]. Recent work using an in vitro pull-down assay indicates that addition of auxin directly to the crude plant extracts promotes the interaction between AUX/IAA and SCF ${ }^{\mathrm{TIR} 1}$. Depletion of membranes from the extract has no effect on the auxin-induced interaction. This result indicates that the auxin receptor and other signaling proteins are soluble factors [41]. Consistent with earlier studies, the interaction is completely abolished if either of the conserved Pro residues in domain II are replaced, suggesting that both conserved Pro residues are important in AUX/IAA-TIR1 interaction. In addition to these insights, this in vitro assay system has been used to address several longstanding questions related to the mechanism of auxin action.

\section{Auxin receptor: still a mystery}

Now that auxin has been shown to function in an extract composed of soluble proteins, the next questions are which proteins are required for the response and, in particular, what is the identity of the auxin receptor. One promising candidate is auxin binding protein 1 (ABP1). ABP1 is known to regulate several physiological processes including tobacco mesophyll protoplast hyperpolarization, cell expansion in tobacco and maize, protoplast division in tobacco mesophyll cells, and stomatal closure [42]. Interestingly, knockout mutants of $A B P 1$ result in embryo lethality, indicating that this gene is essential for plant growth and development [43]. The bulk of the ABP1 is localized to the endoplasmic reticulum, where the $\mathrm{pH}$ is too high for optimal auxin binding. However, a small proportion of ABP1 is on the cell surface and this might function as an extracellular receptor [44]. Although some evidence suggests that $\mathrm{ABP} 1$ is an auxin receptor, so far there are no reports linking ABP1 to auxin-induced gene expression or protein degradation. Moreover, in the in vitro assay described above, neither addition of zmABP1 expressed and purified from insect cells nor depletion of ABP1 from the plant extract using anti-ABP1 antibody

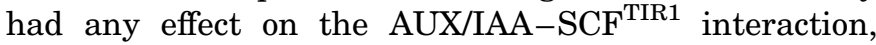
suggesting that ABP1 is not involved in auxin-induced AUX/IAA degradation. However, it is still possible that $\mathrm{ABP} 1$ acts as a receptor for other physiological functions. Additionally, it has been proposed that some auxin transporters possess specialized receptor function [42]. Although this is an interesting hypothesis, recent results indicate that membrane-bound proteins do not function in the signaling cascade leading to AUX/IAA-SCF ${ }^{\text {TIR1 }}$ interaction [41]. At this point, apart from the fact that it is a soluble protein, nothing more is known about the identity of the receptor that mediates auxin-regulated degradation of the AUX/IAA proteins. However, the availability of a biochemical assay for auxin response based on a soluble plant extract should permit rapid progress in this area.

\section{Mechanism of auxin action}

How does auxin promote the interaction between the AUX/IAA proteins and $\mathrm{SCF}^{\mathrm{TIR} 1}$ ? By analogy to other SCF complexes and their substrates, several possible mechanisms have been proposed, including phosphorylation or hydroxylation of AUX/IAA proteins [42,45]. Many known SCF substrates must be phosphorylated to promote their association with the SCF [46]. There are several reports linking phosphorylation to auxin signaling in plants. Mitogen activated protein kinase (MAPK) activity in Arabidopsis is stimulated by auxin and, interestingly, inhibitors that block MAPK activity abolish auxin induced expression of the auxin-responsive reporter BA3::GUS. This suggests that MAPK activity is somehow regulating auxin-induced gene expression [47]. It is also interesting that the recently isolated IBR5 gene encodes a dualspecificity phosphatase; $i b r 5$ mutants are defective in both auxin and ABA signaling [48]. Dual-specificity phosphatases often regulate MAPK pathways, suggesting the involvement of a MAPK in auxin signaling. In another study, mutations in the PINOID Ser/Thr kinase result in defects in auxin response [49]. Phytochrome A is known to phosphorylate the N-terminal half of some AUX/IAA proteins in vitro [50], an event that might regulate auxin-induced AUX/IAA degradation. However, results from several laboratories $[11,41]$ indicate that phosphorylation is not directly involved in regulating AUX/IAA degradation. As discussed above, a 13 amino acid peptide containing domain II is sufficient to confer auxin-induced degradation of luciferase reporter protein [11]. Substitution of potential phosphorylation sites had no effect on stability of the peptide. Moreover, complete removal of ATP from the plant extract or inclusion of the broad range kinase inhibitor staurosporine or the phosphatase inhibitor $\mathrm{NaF}$ had no effect on the auxin induced AUX/IAA$\mathrm{SCF}^{\mathrm{TIR} 1}$ interaction [41]. Collectively, these data strongly suggest that phosphorylation of AUX/IAA or another protein is not required for auxin-dependent AUX/IAA$\mathrm{SCF}^{\mathrm{TIR} 1}$ interaction. However, this does not exclude the possibility that a MAPK, PINOID or phytochrome 
A regulates another step in the auxin-signaling cascade that leads to AUX/IAA degradation.

Prolyl hydroxylation has been found to regulate the interaction between hypoxia-inducible factor (HIF), a human transcription factor, and the ubiquitin protein ligase VCB (pVHL, elongins C and B) [51]. Because domain II contains two conserved prolines known to be required for auxin-dependent SCF recognition, it is tempting to speculate that prolyl hydroxylation plays a role in AUX/ IAA-SCF ${ }^{\text {TIR1 }}$ interaction. However, several inhibitors of prolyl hydroxylase (including dimethyloxalylglycine, $\mathrm{Co}^{2+}$ and dehydro-L-proline) had no effect on the interaction between AUX/IAA and SCF ${ }^{\text {TIR1 }}$, strongly suggesting that prolyl hydroxylation is not involved in this process [41]. Nevertheless, it is still possible that a plant prolyl hydroxylase that is not affected by these inhibitors is involved in this interaction.

Apart from prolyl hydroxylation, there is emerging evidence that prolyl isomerization is involved in signal transduction pathways [52]. Peptidyl-prolyl cis-trans isomerases (PPIases or rotamases) are a group of enzymes that catalyse the cis-trans isomerization of the peptide bond $\mathrm{N}$-terminal to a proline residue in proteins. This is especially true when the residue preceding the proline is a bulky group. Based on inhibitor specificity and sequence similarity, there are three groups of PPIases, known as cyclophilins, FK506-binding proteins (FKBPs) and parvulins [52]. Cyclophilins, FKBPs and parvulins are inhibited by cyclosporine A (CsA), FK506 and juglone, respectively. There is no sequence or structural similarity between these three groups of PPIases.

The role of PPIases in auxin response was assessed using the in vitro assay. Neither cyclosporine-A nor rapamycin had any effect on AUX/IAA-SCF ${ }^{\mathrm{TIR} 1}$ interaction but juglone dramatically inhibited auxin-induced interaction. Similarly, juglone inhibited auxin-induced expression of BA3::GUS in seedlings as well as auxininduced degradation of AXR3NT-GUS, suggesting that the parvulin type of PPIase might be involved in auxin signaling [41] (Figure 2). If this is the case, it is possible that cis-trans isomerization within domain II of AUX/IAA proteins in response to auxin might be the trigger for

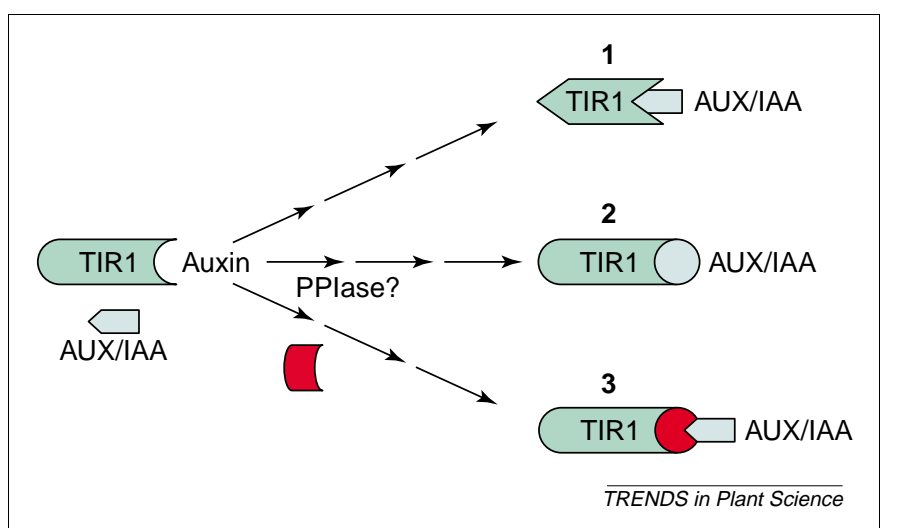

Figure 2. Models to explain auxin-induced AUX/IAA-TIR1 interaction. Auxin might induce modifications in TIR1 (1) or in domain II of AUX/IAA proteins (2), possibly through cis-trans isomerization involving peptidyl-prolyl cis-trans isomerases (PPlases). Alternatively, auxin might modify an adaptor protein that is required for AUX/IAA-TIR1 interaction (3). auxin-induced interaction between AUX/IAA and $\mathrm{SCF}^{\mathrm{TIR} 1}$. Alternatively, PPIases might act on another protein, perhaps TIR1, in response to auxin (Figure 2). However, it should also be noted that juglone is not a specific inhibitor of PPIases. Thus, it is possible that juglone inhibits the AUX/IAA-SCF ${ }^{\text {TIR1 interaction }}$ through a different mechanism and that PPIases are not involved in the interaction. There are three genes in the Arabidopsis genome that encode parvulins (At2 g18040, At5 g19370 and At1 g26550). Further studies will be required to determine whether any of these proteins are important for the auxin response.

Parvulins have also been implicated, albeit indirectly, by the results of a chemical genetics approach to auxin action. Yunde Zhao et al. identified and isolated a novel mutant called sir 1 that is resistant to a compound called sirtinol, which is a small molecule that mimics auxin activity, including induction of auxin-regulated genes and promotion of auxin-related developmental phenotypes [53]. Similar to auxin treatment, sirtinol (an inhibitor of the sirtuin family of nicotinamide-adenine-dinucleotidedependent deacetylases) induces rapid degradation of AXR3NT-GUS reporter fusion protein, indicating that sirtinol-induced gene expression might require regulated protein degradation [53]. Because sirtinol appears to act like an auxin and the sir 1 mutant is resistant to sirtinol, one might expect the mutant also to have an altered response to auxin. This is the case, but not in the expected way. Instead of displaying resistance to auxin, the sir 1 mutant is hypersensitive to the hormone. Because $\operatorname{sir} 1$ is recessive, this results suggest that SIR1 is a negative regulator of auxin response. How this relates to sirtinol resistance is not clear at present. Analysis of the domain architecture of SIR1 reveals the presence of an E1-like domain (similar to AXR1) in its N-terminus and a rhodanese-like domain at the C-terminus. This domain was originally identified in rhodanese proteins and later identified in a wide range of proteins [54]. Interestingly, one of the three parvulin type of prolyl isomerases also contains a rhodanese-like domain in its C-terminus.

Taking into account the current state of our knowledge, it is possible that the increased levels of auxin promote the interaction between AUX/IAA and TIR1 through a modification of either TIR1 (Figure 2a) or of domain II of the AUX/IAA (Figure 2b). It is also possible that auxin causes a modification of an adaptor protein that is required for AUX/IAA-TIR1 interaction (Figure 2c). Whether these modifications are mediated through a PPIase or another activity remains to be determined.

\section{Conclusions}

During the past few decades, much progress has been made in elucidating the auxin signaling pathway. Obviously, there is still much to learn. Perhaps the most important outstanding question is the identity of the auxin receptor(s). We also need to understand how the large families of AUX/IAA and ARF proteins regulate downstream gene expression. Given the extent of their sequence identity, many AUX/IAA and ARFs are likely to be functionally redundant. It will be interesting to know how these two groups of proteins act coordinately to 
regulate a diverse array of genes during the growth and development of plants. Finally, apart from the primary auxin response genes mentioned earlier, we know little about the downstream genes that mediate auxin response. Several groups have used microarrays to characterize the auxin-induced transcriptome in Arabidopsis seedlings [55, $56]$. Further studies of the genes identified in this way will be essential to our understanding of how auxin regulates growth and development.

\section{Acknowledgements}

Work in our laboratory was supported by grants from the NIH (43644), NSF (DBI 0115870) and DOE (DE-FG02-02ER15312) to M.E.

\section{References}

1 Pennazio, S. (2002) The discovery of the chemical nature of the plant hormone auxin. Riv. Biol. 95, 289-308

2 Abel, S. and Theologis, A. (1996) Early genes and auxin action. Plant Physiol. 111, 9-17

3 Ulmasov, T. et al. (1997) Aux/IAA proteins repress expression of reporter genes containing natural and highly active synthetic auxin response elements. Plant Cell 9, 1963-1971

4 Guilfoyle, T. et al. (1998) How does auxin turn on genes? Plant Physiol. $118,341-347$

5 Guilfoyle, T.J. and Hagen, G. (2001) Auxin response factors. J. Plant Growth Regul. 10, 281-291

6 Liscum, E. and Reed, J.W. (2002) Genetics of AUX/IAA and ARF action in plant growth and development. Plant Mol. Biol. 49, 387-400

7 Ulmasov, T. et al. (1999) Activation and repression of transcription by auxin response factors. Proc. Natl. Acad. Sci. U. S. A. 96, 5844-5849

8 Ulmasov, T. et al. (1999) Dimerization and DNA binding of auxin response factors. Plant J. 19, 309-319

9 Tiwari, S.B. et al. (2003) The roles of auxin response factor domains in auxin-responsive transcription. Plant Cell 15, 533-545

10 Hellmann, H. and Estelle, M. (2002) Plant development: regulation by protein degradation. Science $297,793-797$

11 Ramos, J.A. et al. (2001) Rapid degradation of AUX/IAA proteins requires conserved amino acids of domain II and is proteasomedependent. Plant Cell 13, 2349-2360

12 Gray, W.M. et al. (2001) Auxin regulates $\mathrm{SCF}^{\mathrm{TIR} 1}$-dependent degradation of AUX/IAA proteins. Nature 414, 271-276

13 Ouellet, F. et al. (2001) IAA17/AXR3: biochemical insight into an auxin mutant phenotype. Plant Cell 13, 829-841

14 del Pozo, J.C. et al. (1998) The ubiquitin-related protein RUB1 and auxin response in Arabidopsis. Science 280, 1760-1763

15 Gray, W.M. et al. (1999) Identification of an SCF ubiquitin-ligase complex required for auxin response in Arabidopsis thaliana. Genes Dev. 13, 1678-1691

16 Gagne, J.M. et al. (2002) The F-box subunit of the SCF E3 complex is encoded by a diverse super family of genes in Arabidopsis. Proc. Natl. Acad. Sci. U. S. A. 99, 11519-11524

17 Gray, W.M. et al. (2002) Role of the Arabidopsis RING-H2 protein RBX1 in RUB modification and SCF function. Plant Cell 14, $2137-2144$

18 Hellmann, H. et al. (2003) AXR6 encodes CUL1 implicating SCF ubiquitin ligases in auxin regulation of embryogenesis. EMBO J. 22 , $3314-3325$

19 Rouse, D. et al. (1998) Changes in auxin response from mutations in an AUX/IAA gene. Science 279, 1371-1373

20 Tian, Q. and Reed, J. (1999) Control of auxin-regulated root development by Arabidopsis thaliana SHY/IAA3 gene. Development $126,711-721$

21 Nagpal, P. et al. (2000) AXR2 encodes a member of the AUX/IAA protein family. Plant Physiol. 123, 563-573

22 Rogg, L.E. et al. (2001) A gain-of-function mutation in IAA28 suppresses lateral root development. Plant Cell 13, 465-480

23 Zenser, N. et al. (2003) Acceleration of AUX/IAA proteolysis is specific for auxin and independent of AXR1. Plant J. 35, 285-294

24 Tian, Q. et al. (2003) Regulation of Arabidopsis SHY2/IAA3 protein turnover. Plant J. 36, 643-651

25 Dharmasiri, S. et al. (2003) The RUB/NEDD8 conjugation pathway is required for early development in Arabidopsis. EMBO J. 22, $1762-1770$

26 Kamura, T. et al. (1999) The Rbx1 subunit of SCF and VHL E3 ubiquitin ligase activates Rub1 modification of cullins Cdc53 and Cul2. Genes Dev. 13, 2928-2933

27 Hamann, T. et al. (2002) The Arabidopsis BODENLOS gene encodes an auxin response protein inhibiting MONOPTEROS-mediated embryo patterning. Genes Dev. 16, 1610-1615

28 Galan, J. and Peter, M. (1999) Ubiquitin dependant degradation of multiple F-box proteins by an autocatalytic mechanism. Proc. Natl. Acad. Sci. U. S. A. 96, 9124-9129

29 Mathias, N. (1999) The abundance of cell cycle regulatory protein Cdc4p is controlled by interactions between its F box and Skp1p. Mol. Cell. Biol. 19, 1759-1767

30 Morimoto, M. et al. (2000) Modification of cullin-1 by ubiquitin like protein NEDD8 enhances the activity of $\mathrm{SCF}^{\text {skp2 }}$ towards p2 $7^{\mathrm{kip} 1}$. Biochem. Biophys. Res. Commun. 270, 1093-1096

31 Read, M.A. et al. (2000) NEDD8 modification of cul-1 activates $\mathrm{SCF}\left(\right.$ beta $\left.^{\mathrm{TrCP}}\right)$ - dependant ubiquitination of IkappaBalpha. Mol. Cell. Biol. 20, 2326-2333

$32 \mathrm{Wu}, \mathrm{K}$. et al. (2000) Conjugation of NEDD8 to CUL1 enhances the ability of the ROC1-CUL1 complex to promote ubiquitin polymerization. J. Biol. Chem. 275, 32317-32324

33 Kawakami, T. et al. (2001) NEDD8 recruits E2-ubiquitin to SCF E3 ligase. EMBO J. 20, 4003-4012

34 Pintard, L. et al. (2003) NEDDylation and deNEDDylation of CUL3 is required to target ME1-1/Katanin for degradation at the meiosis-tomitosis transition in C. elegans. Curr. Biol. 13, 911-921

35 Schwechheimer, C. et al. (2001) Interactions of the COP9 signalosome with the E3 ubiquitin ligase $\mathrm{SCF}^{\mathrm{TIR} 1}$ in mediating auxin response. Science 292, 1379-1382

36 Yang, X. et al. (2002) The COP9 signalosome inhibits p27 $7^{\mathrm{kip} 1}$ degradation and impedes $\mathrm{G} 1-\mathrm{S}$ phase progression via deNEDDylation of SCF ${ }^{\text {Cul1 }}$. Curr. Biol. 12, 667-672

37 Wei, N. et al. (1994) Arabidopsis COP9 is a component of a novel signaling complex mediating light control of development. Cell 78, $117-124$

38 Liu, J. et al. (2002) NEDD8 modification of CUL1 dissociates p120 ${ }^{C A N D 1}$, an inhibitor of CUL1-SKP1 binding and SCF ligases. Mol. Cell 10, 1511-1518

39 Zheng, J. et al. (2002) CAND1 binds to unNEDDylated CUL1 and regulates the formation of SCF ubiquitin E3 ligase complex. Mol. Cell $10,1519-1526$

40 Oshikawa, K. et al. (2003) Preferential interaction of TIP120A with Cul1 that is not modified by NEDD8 and not associated with Skp1. Biochem. Biophys. Res. Commun. 303, 1209-1216

41 Dharmasiri, N. et al. (2003) Auxin action in a cell-free system. Curr. Biol. 13, 1418-1422

42 Kepinski, S. and Leyser, O. (2002) Ubiquitination and auxin signaling: a degrading story. Plant Cell 14, S81-S95

43 Chen, J.G. et al. (2001) ABP1 is required for organized cell elongation and division in Arabidopsis embryogenesis. Genes Dev. $15,902-911$

44 Henderson, J. et al. (1997) Retention of maize auxin-binding protein in the endoplasmic reticulum: quantifying escape and the role of auxin. Planta 202, 313-323

45 Dharmasiri, S. and Estelle, M. (2002) The role of regulated protein degradation in auxin response. Plant Mol. Biol. 49, 401-409

46 Gray, W.M. and Estelle, M. (2000) Function of the ubiquitinproteosome pathway in auxin response. Trends Biochem. Sci. 25, $133-138$

47 Mockaitis, K. and Howell, S.H. (2000) Auxin induces mitogenic activated protein kinase (MAPK) activation in roots of Arabidopsis seedlings. Plant J. 24, 785-796

48 Augustus, M.M. et al. (2003) IBR5, a dual-specificity phosphatase-like protein modulating auxin and abscisic acid responsiveness in Arabidopsis. Plant Cell 15, 2979-2991

49 Christensen, S.K. et al. (2000) Regulation of auxin response by the protein kinase PINOID. Cell 100, 469-478

50 Colon-Carmona, A. et al. (2000) AUX/IAA proteins are phosphorylated by phytochrome in vitro. Plant Physiol. 124, 1728-1738

$51 \mathrm{Kim}$, W. and Kaelin, W.G. (2003) The von Hippel-Lindau tumor 
suppressor protein: new insights into oxygen sensing and cancer. Curr Opin. Genet. Dev. 13, 55-60

52 Harrar, Y. et al. (2001) FKBPs: at the crossroads of folding and transduction. Trends Plant Sci. 6, 426-430

53 Zhao, Y. et al. (2003) SIR1, an upstream component in auxin signaling identified by chemical genetics. Science 301, 1107-1110

54 Bordo, D. and Bork, P. (2002) The rhodanese/Cdc25 phosphatase superfamily. Sequence-structure-function relations. EMBO Rep. 3, 741-746

55 Qing, T. et al. (2002) Arabidopsis SHY2/IAA3 inhibits auxin-regulated gene expression. Plant Cell 14, 301-319

56 Pufky, J. et al. (2003) The auxin-induced transcriptome for etiolated Arabidopsis seedlings using a structure/function approach. Funct. Integr. Genomics 3, 135-143

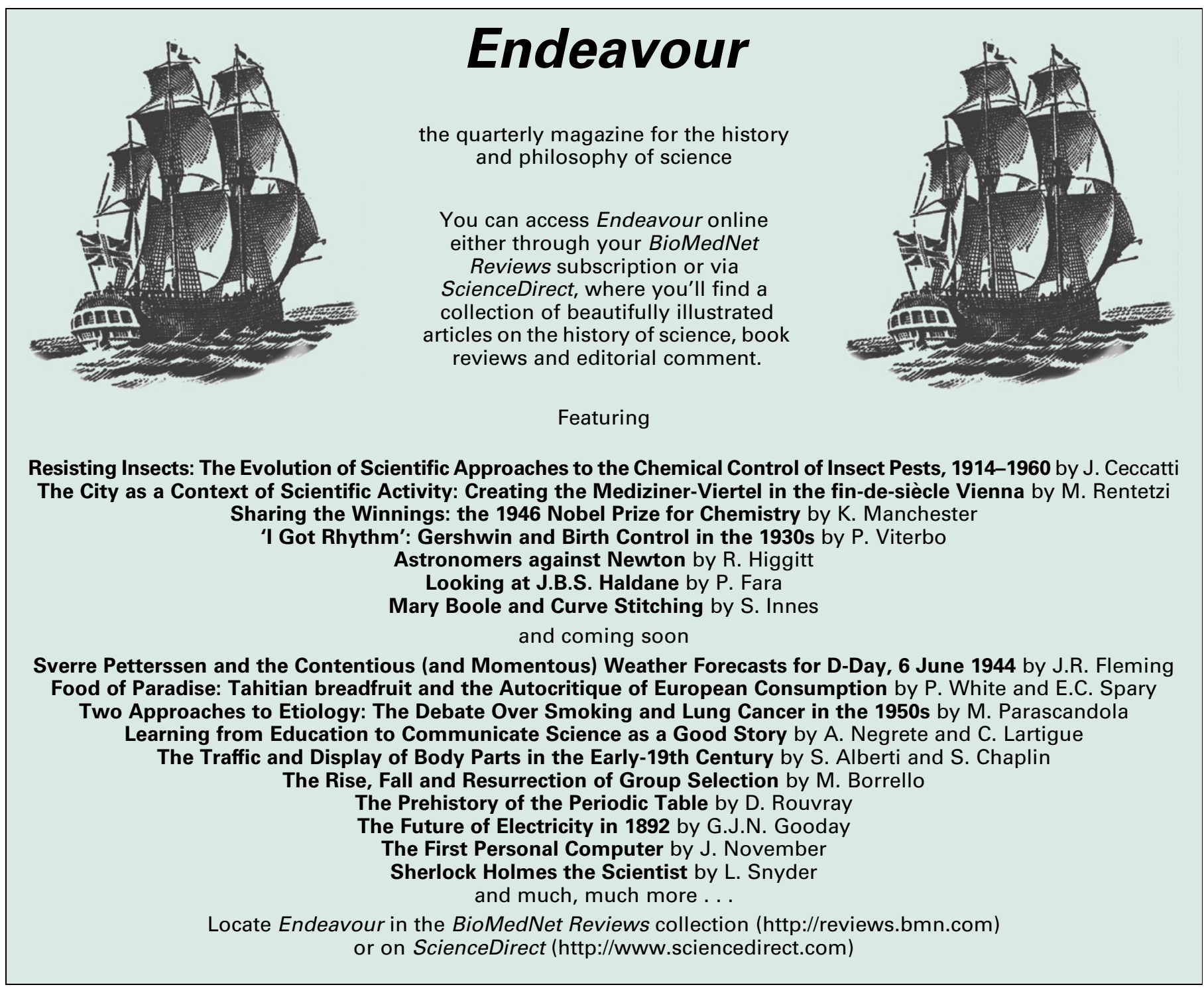

